|| ISSN(online): 2589-8698 || ISSN(print): 2589-868X ||

International Journal of Medical and Biomedical Studies

Available Online at www.ijmbs.info

NLM (National Library of Medicine ID: 101738825)

Index Copernicus Value 2019: 79.34

Case Report

Volume 5, Issue 1; January: 2021; Page No. 100-102

\title{
A MENKES DISEASE CASE WITH MULTIPLE COMORBIDITIES
}

Dr. Salha Aljohani, ${ }^{1,}$ Abdullah Saleh Alfayez ${ }^{2}$, Abdullah Abdulaziz Khojah ${ }^{2}$, Mohamad S. Alsamal ${ }^{2}$, Muhanad Mansour Almekbel ${ }^{2}$, Mohammed Abdulaziz Khojah ${ }^{2}$, Ahmed Mohammed AlJohani ${ }^{3}$, Mohammed Nidal Malhis ${ }^{4}$, Tasnim Nidal Malhis ${ }^{5}$

${ }^{1}$ A Paediatric Consultant in Maternity and Children Hospital, Medina, Saudi Arabia.

${ }^{2}$ College of Medicine, Sulaiman AlRajhi University, Al Bukairiyah, Al Qassim, Saudi Arabia.

${ }^{3}$ Taibah College of Medicine, Taibah University, Al-Madinah Al-Munawwarah, Saudi Arabia.

${ }^{4}$ Almaarefa University, Ad Diriyah, Riyadh, Saudi Arabia.

${ }^{5}$ Faculty of Medicine, University of Aleppo, Aleppo, Syria.

Article Info: Received 08 December 2020; Accepted 08 January 2021

DOI: https://doi.org/10.32553/ijmbs.v5i1.1638

Corresponding author: Dr. Salha Aljohani

Conflict of interest: No conflict of interest.

\section{Abstract}

We report our case of a 15 months old male child, a product of nonconsanguineous marriage presented with increased seizure frequency more than 4 times a day with fever and upper respiratory tract infection for 1 month. He diagnosed with Menkes disease and his oldest brother died at age of 5 year due to the same disease. Menkes disease is a rare congenital disorder of copper metabolism with severe multisystemic manifestations that are primarily characterized by progressive neurodegeneration and connective tissue anomalies. He had almost the typical clinical finding but also, he had other complications which are non commonly found in such cases. As the Menkes disease is incurable disease, early diagnosis and parental counseling is very important for the mother in order to prevent getting a child with same disease.

\section{Introduction}

Menkes disease (MD) is a progressive rare copper metabolism multisystemic disease. It exhibits X-linked recessive pattern of inheritance. As expected, most of the patients are male, but few female patients have been reported. It is most likely due to $\mathrm{X}$ autosome translocation where normal $\mathrm{X}$ is preferentially inactivated. ${ }^{(1)}$

The basis of MD is mutations that have been detected in ATP7A gene which is a transmembrane protein that is responsible for transport of copper and distribution through body cells. ${ }^{\text {(2) (3) }}$ It is positioned in the q arm of chromosome $\mathrm{X}$ at $\mathrm{Xq} 21.1$. Those mutations include deletion, partial deletion, insertion, and missense mutations; with more than 350 mutations have been identified. ${ }^{(3)}$ Abnormal excessive accumulation of copper in enterocytes due to failure of ATP7A efflux pump which leads to this accumulation. This excessive accumulation in enterocytes causes generalized Copper deficiency in body tissues. ${ }^{(4)}(5)$ Copper is essential for many biochemical processes such as cytochrome $\mathrm{C}$ oxidase, lysyl oxidase, tyrosinase and dopamine betahydroxylase. Central nervus system disturbances and autonomic instability are caused by abnormal dopamine beta-hydroxylase activity due to deficiency. Other systemic involvement is connective tissue abnormalities which occurs due to impaired lysyl oxidase activity. Other systemic involvement includes Skin and Hair abnormalities. (6)

Classical Menkes disease patients usually exhibit severe clinical course with death in early childhood typically by three years. Progressive neurodegeneration and connective tissue dysfunction characterize the clinical picture of classical MD.

Pregnancy and labor are usually uncomplicated with normal anthropometry at birth. Clinical features in the newborn period include prolonged jaundice, hypothermia, hypotonia, and feeding difficulties at birth ${ }^{(7)}$. Spontaneous fractures and cephalohematoma may present at birth. The typical symptoms usually start by two months with characteristic skin and hair findings. Hair is normal at birth but is replaced by fine, sparse kinky hair. Sagging facial features, micrognathia, cutis laxa, and blue iris are noted ${ }^{(7)}$. Various congenital malformations have been reported, including congenital microblepharia, entropion, long arched palate, cerebellar hypoplasia, cystic changes in the lungs, and complete AV block ${ }^{(7)}{ }^{(8)}$. Neurological manifestations are the most common and characteristic of Menkes disease. Developmental regression and seizures are usually the first to start around 2 to 3 months. Infection or febrile state triggers the onset of seizures. The disease can present in other ways like nonconvulsive seizures and recurrent episodes of apnea. ${ }^{(9)}$

Abnormalities in connective tissue are very common in MD. This includes: skin, muscles and bone deformities all over the body. (10) (11) Ocular abnormalities include poor visual acuity, aberrant eyelashes and retinal disorders. ${ }^{(11)}$ (10)

The diagnosis of Menkes disease is made by the characteristic clinical, biochemical, and radiological features. Biochemical testing through measuring serum 
copper level which is suspected to be low in MD. The restriction of copper serum lever testing is that it is usually low in infant less than 6 months. So, it should be combined with another testing. Clinical evaluation plays a major role in diagnosis. ${ }^{(12)(13)}$

Neuroradiology also has a role in diagnosis. cerebral atrophy and delayed myelination are the most commonly observed features on the MRI brain.) Other features include subdural effusion, hematoma and hygroma, leukoencephalopathy, basal ganglia signal changes, and intracranial vessel tortuosity, focal tumefactive white matter lesions on MRI are especially seen before ten months of age. Hyperintense drop-shaped lesions in centrum semiovale, with restricted diffusion, also have been reported $^{(12)(13)}$.

Lines of treatment are mainly symptomatic and supportive. This includes Copper injections, Anticonvulsants, proper feeding and Physiotherapy. ${ }^{(14)}$

\section{Case presentation:}

A 15 months old male child, a product of nonconsanguineous marriage presented with increased seizure frequency more than 4 times a day with fever and upper respiratory tract infection for 1 month.

The child seizure disorder started at 4 months of age, he had history of recurrent admissions for same complaint. He also had global development delay, swallowing difficulty, hypotonia and known to have neurogenic bladder.

Patient discovered to have hiatal hernia, a fundoplication was done for him and he became stable.

The child was born as term baby without any significant antenatal and postnatal complication. He was on formula feeding without any complication. The child has a sister with normal development and she did not have any health issues, his older brothers was diagnosed as Menkes disease and he passed away at age of 5 years as a result of this disease.

On examination the baby looked ill and had a global developmental delay, sagging face and very typical kinky hair. Other systemic examination is unremarkable.

Investigation showed normal CBC, electrolytes, Liver function test, thyroid function and Vit D. However, urine organic acid showed increased ketone body. Metabolic screening was unremarkable. Serum ceruloplasmin and copper were low. Upper and lower abdominal ultrasounds were normal. Genitourinary US revealed undescended testis.

EEG showed multifocal sharp spikes and slow wave discharges more on the right posterior quadrant. No seizure was recorded during EEG. The slow background activity indicates cortical dysfunction.

MRI brain and MRA showed diffuse generalized cerebral and cerebellar brain atrophy with abnormal T2 signal intensity in bifrontal region. Moreover, brainstem, basal ganglia and thalami were minimally small. In addition, elongated tortuous vessels with dysmyelination of frontal white matter were noticed raising the possibility of Menkes disease.

Menkes disease genetic test was done for the child and mother by Whole Exome Sequencing and results to be released.

The child now being admitted and treated symptomatically in children and maternity hospital.

\section{Discussion:}

Menkes disease is a rare X-linked recessive inherited multisystemic disease. Males are more affected than females. A triad of developmental delay, neurological degeneration and hair abnormalities are characteristic for this disease. It is usually manifesting at the age of 2-3 month and patients usually die at 3-4 years of age due to pneumonia. ${ }^{(16)}$

A specific care should be taking in order to treat the patient and to prevent mother from getting other siblings with same disease.

For the first issue, a baby should be treated with multidisciplinary team. In our case, the patient was admitted many times due to seizure episodes and fever and also failure to thrive without unknown cause in the begging except for the general effect of this disease, and after taking more detailed history about this new complain, the mother said that his child has difficulty in the swallowing during feeding and the milk was come out from the mouth, and after doing the appropriate investigation we discovered that the patient have hiatal hernia, a surgical intervention was done (fundoplication) and now the patient became better in feeding, so multidisciplinary team is critical in such cases .

in term of diagnosis and the clinical features of this disease, our case had an additional clinical feature which are hiatal hernia and the neurogenic bladder, and these are not common clinical finding in such cases, and this contributes in rising awareness in pediatrician about the atypical presentations in the rare diseases. About the diagnosis, we diagnosed the patient by genetic testing and this was done for the child and mother, and by biochemical testing through measuring serum copper level, and this are the gold stander of diagnosis. Moreover, a family history did a crucial role in diagnosis since the patient's mother had a child with same disease.

We have managed this patient symptomatically using multidisciplinary approach. Although, this disease occurs due to cupper deficiency, copper supplementation is not effective. This is because copper is absorbed poorly in the gastrointestinal tract, the brain-blood barrier prevents copper delivery. ${ }^{(17)}$ However, parenteral copper histidine might be beneficial when given early in the course of the disease. $^{(18)}$ 
Menkes disease is incurable disease, thus early diagnosis and parental counseling is the cornerstone to reduce the complications and mortality.

Prenatal diagnosis of Menkes disease can be done using genetic analysis or measurement of the copper concentration in amniotic fluid and chorionic villi cells. ${ }^{(19)}$ Thus, early diagnosis of this condition can help in genetic counselling.

\section{Conclusion:}

A 15 months old male child, a product of nonconsanguineous marriage presented with increased seizure frequency more than 4 times a day with fever and upper respiratory tract infection. He diagnosed as Menkes disease child, He had multilabel comorbidities and his oldest brother died due to the same disease. Early diagnosis and parental counseling are the cornerstone to reduce the morbidity and mortality in this patient.

\section{References:}

1. Tümer Z, Møller LB. Menkes disease. Eur J Hum Genet. 2010;18(5):511-518. doi:10.1038/ejhg.2009.187

2. Kaler SG, Ferreira CR, Yam LS. Estimated birth prevalence of Menkes disease and ATP7A-related disorders based on the Genome Aggregation Database (gnomAD). Mol Genet Metab Rep. 2020;24:100602. Published 2020 Jun 5. doi:10.1016/j.ymgmr.2020.100602

3. Scheiber I, Dringen R, Mercer JF. Copper: effects of deficiency and overload.Met Ions Life Sci. 2013;13:359-387. doi:10.1007/978-94-007-7500$8+11$

4. Schmidt K, Ralle M, Schaffer T, et al. ATP7A and ATP7B copper transporters have distinct functions in the regulation of neuronal dopamine- $\beta$ hydroxylase. J Biol Chem. 2018;293(52):2008520098. doi:10.1074/jbc.RA118.004889

5. Steveson TC, Ciccotosto GD, Ma XM, Mueller GP, Mains RE, Eipper BA. Menkes protein contributes to the function of peptidylglycine alpha-amidating monooxygenase. Endocrinology. 2003;144(1):188200. doi:10.1210/en.2002-220716

6. Ojha R, Prasad AN. Menkes disease: what a multidisciplinary approach can do. $J$ Multidiscip Healthc. 2016;9:371-385. Published 2016 Aug 17. doi:10.2147/JMDH.S93454

7. Hicks JD, Donsante A, Pierson TM, Gillespie MJ, Chou DE, Kaler SG. Increased frequency of congenital heart defects in Menkes disease. Clin
Dysmorphol. 2012;21(2):59-63. doi:10.1097/ MCD. 0b013e32834ea52b

8. Verrotti A, Cusmai R, Darra F, et al. Epilepsy in Menkes disease: an electroclinical long-term study of 28 patients. Epilepsy Res. 2014;108(9):15971603. doi:10.1016/j.eplepsyres.2014.08.006

9. Gasch AT, Caruso RC, Kaler SG, Kaiser-Kupfer M. Menkes' syndrome: ophthalmic findings. Ophthalmology. 2002;109(8):1477-1483. doi:10.1016/s0161-6420(02)01095-3

10. Moon SJ, An YM, Kim SK, Kwon YS, Lee JE. The effect of low-dose intravenous bisphosphonate treatment on osteoporosis in children with quadriplegic cerebral palsy. Korean J Pediatr. 2017;60(12):403-407.

doi:10.3345/kjp.2017.60.12.403

11. Manara R, D'Agata L, Rocco MC, et al. Neuroimaging Changes in Menkes Disease, Part 1. AJNR Am J Neuroradiol. 2017;38(10):1850-1857. doi:10.3174/ajnr.A5186

12. Manara R, Rocco MC, D'agata L, et al. Neuroimaging Changes in Menkes Disease, Part 2. AJNR Am J Neuroradiol. 2017;38(10):1858-1865. doi:10.3174/ajnr.A5192

13. Tønnesen $\mathrm{T}$, Kleijer WJ, Horn N. Incidence of Menkes disease. Hum Genet. 1991;86(4):408-410. doi:10.1007/BF00201846

14. Kaler SG, Holmes CS, Goldstein DS, et al. Neonatal diagnosis and treatment of Menkes disease. $N$ Engl J Med. doi:10.1056/NEJMoa070613

15. Aicardi J. Menkes disease (kinky hair disease, steely hair disease, trichopoliodystrophy). In Aicardi J (Ed). Diseases of the nervous system in childhood (second edition). London: Mac Keith Press, 1998:306-

16. Datta AK, Ghosh T, Nayak K, Ghosh M. Menkes kinky hair disease: A case report. Cases J. 2008;1(1):158. Published 2008 Sep 18 doi:10.1186/1757-1626-1-158

17. Kodama H, Murata Y, Kobayashi M. Clinical manifestations and treatment of Menkes disease and its variants. Pediatr Int. 1999;41(4):423-429. doi:10.1046/j.1442-200x.1999.01095.x

18. Christodoulou J, Danks DM, Sarkar B, et al. Early treatment of Menkes disease with parenteral copperhistidine: long-term follow-up of four treated patients. Am J Med Genet. 1998;76(2):154-164.

19. $\mathrm{Gu}$ YH, Kodama H, Sato E, et al. Prenatal diagnosis of Menkes disease by genetic analysis and copper measurement. Brain Dev. 2002;24(7):715-718. doi:10.1016/s0387-7604(02)00093-1 